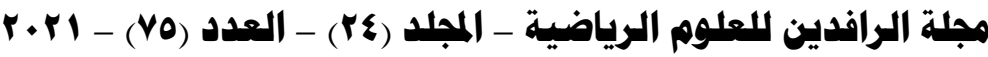

دراسة تحليلية للعلوم الأساسية ذات الأولوية في إعداد وتأهيل المدرب الرياضي

دراسة تصليلية للعلوم الأساسية ذات الأولوية في إعداد وتأهيل

\section{المدرب الرياضي}

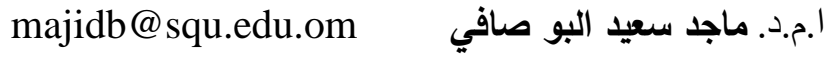

قسم التربية البذنية وعلوم الرياضة/ كلية التربية/ جامعة السلطان قابوس صلئ

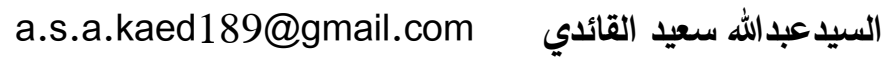

وزارة التربية والتعليم/ العديرية العامة للتربية والتعليم/ مسقط

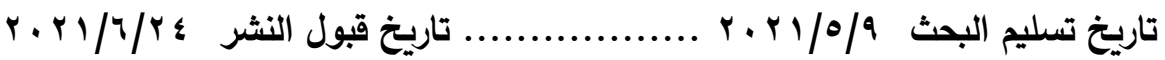

DOI:

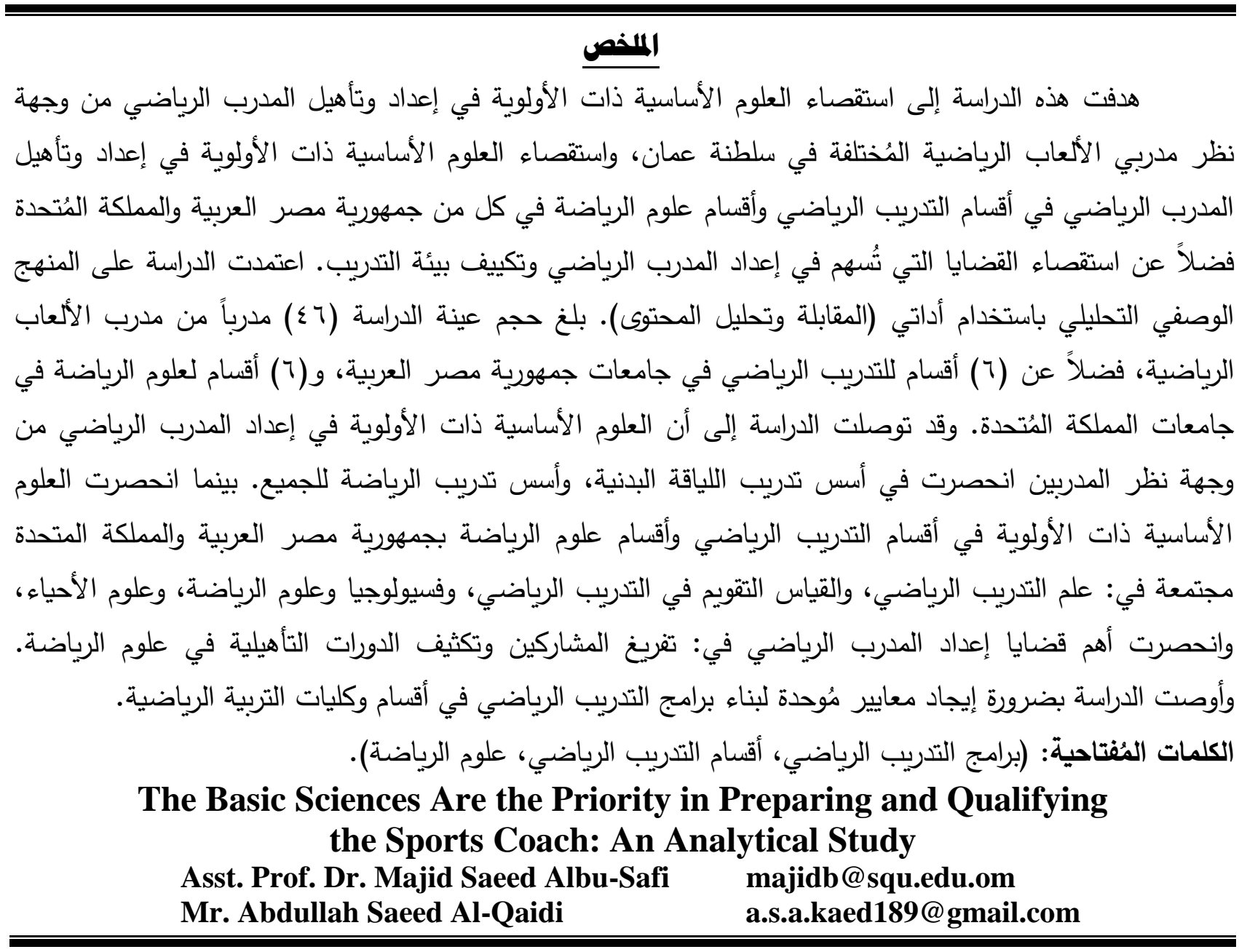

\section{ABSTRACT}

This study aimed to investigate the basic sciences in preparing and qualifying sports coaches from the viewpoint of the various sports coaches in the Sultanate of Oman, and investigating the basic sciences in preparing and qualifying a sports coach in the sports training departments and the sports science departments in both the Arab Republic of Egypt and the United Kingdom. This study also aimed to investigate the issues that contribute to preparing sports coaches and adapting the training environment. The study relied on the descriptive and analytical method by using (interview and content analysis). The study sample reached (46) trainers a sports coaches, in addition, (6) departments for sports training in the universities of the Arab Republic of Egypt and (6) departments for sports science in the universities of the United Kingdom. The study concluded that the basic sciences in preparing sports coaches from the coaches' viewpoint are confined to the foundations of fitness training and the foundations for training sports for all. While the basic 


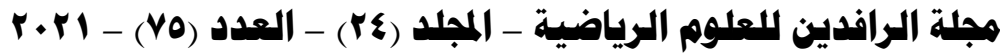

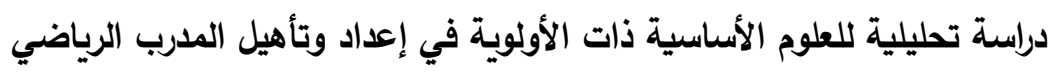

sciences with priority were confined to the sports training departments and the sport sciences departments in the Arab Republic of Egypt and the United Kingdom combined in: the science of sports training, measurement and assessment in sports training, physiology and science of sports, biology. The most important issues of preparing a sports coaches was intensifying rehabilitation courses in sports science. The study recommended the necessity of creating unified standards for building sports training programs in departments and colleges of physical education.

Keywords: (Sports Training Programs, Departments of Sports Training, Sports Science).

\section{1- التعريف بالدراسة}

\section{1- المقدمة وأهمية الدراسة}

Lara-Bercial et ) يؤدي المدربين دوراً رئيساً في تعزيز المشاركة الرياضية وتعزيز أداء الفرق والرياضيين

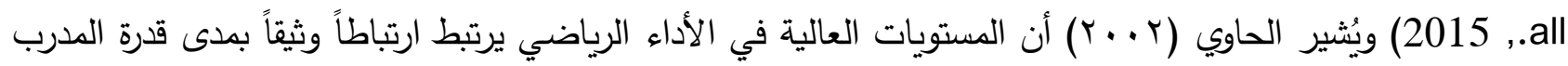
على إدارة وتتفيذ العملية التدريبية، لذا فإن الوصول بالمدرب إلى المستوى الفني الذي يؤهله لقيادة المتدربين والوصول

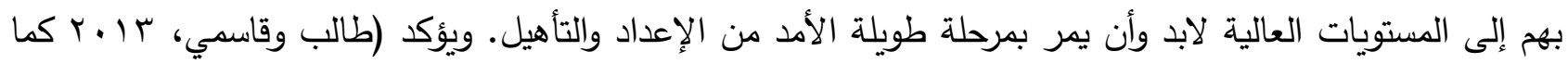
ورد في Mohammadi \& Azizi, 2019) أن التدريب هو عملية تعليم وتدريب مصممة لتحسين أداء الإنسان وتطوره من خلال النشاط البدني. ومن ثم، فإن تحقيق هذا الهدف الجدير بالاهتمام لا يمكن تحقيقه إلا من خلال صياغة برامج منهجية هادفة، ويُشير كروكثانك وكولينز (Cruickshank \& Collins, من (إنى 2015) إلى أن الدور الأساسي للمدرب الرياضي هو تطوير وتحسين أداء الأفراد والفرق، فضلاً عن الاتصال الدائم مع المتدربين خارج بيئة التدريب المباشر . بدأ المدرب الرياضي بممارسة مهامه لقيادة اللاعبين منذ فترة زمنية طويلة، فعلى المستوى العالمي مثالاً فإن دورة الألعاب الاولمبية والتي بدأت أولى دوراتها في 1971 قاد مجموعة من المدربين لاعبيهم للمشاركة في الدورة الأولمبية الأولى، ومهما يكن من أمر، فإن المهام التي كان يقوم بها المدرب في تلك الفترة الزمنية تختلف اختلافاً كبيراً عن متطلبات ومؤهلات المدرب الرياضي الذي استعد لمشاركة لاعبيه في أولمبياد طوكيو اY ·.T، ويشهد علم التدريب الرياضي دخول الأدوات الرياضية والأجهزة التكنولوجية في أساس العملية التدريبية وبشكل مستمر • ويُشير جانو اوفرواي وآخرون (Gano-overway et all)., 2020) أنه ومع استمرار نمو مهنة التدريب الرياضي، هناك حاجة للتفكير في Taylor, B. مراجعة المعارف والكفاءات التي يجب أن يمتلكها المدربون لدعم الخبرات الرياضية. ويرى تايلور وجرات \& Garratt مدرباً مؤهلاً لخوض عملية التدريب، وتتركز أهمية المدرب الرياضي في قدرته على صقل وتطوير القدرات البدنية والمهارية والخططية للاعبين. وقدرته في الحفاظ على تماسك ووحدة المتدربين، وكذلك غرس القيم التربوية وتعزيز

$$
\text { التوازن النفسي تحت ضغوط الأحداث الرياضية. }
$$

وتتأثر مهام ومؤهلات المدرب الرياضي بعدد من العوامل بحسب ما تقتضيه المهمة التدريبية وأهداف البرنامج

التدريبي، إذ يُشير لارا بارسيال( Lara-Bercial et all., 2015) أن لخبرة المدرب دور في تكوين وبناء العملية التدريبية ومن ثم فان نتاج المدرب الخبير هي في الغالب أكثر وضوحاً من نتاج المدرب الجديد، ويُشير سوروجلال وآخرون (Surujlal., et all, وسلوك الأداء وذلك في دراسته التي استهدفت تقييم أداء المدربين في كل من جنوب أفريقيا وكندا، وتتمثل أهمية دراسة العوامل المؤثرة في العملية التدريبية لمعرفة فيما إذا كانت هناك عوامل مصاحبة تؤثر على أساس العملية التدريبية 
بالسلب أو بالإيجاب، وبالتالي الععل على تحييد العوامل السلبية منها، ودعم العوامل الإيجابية بما يخدم عملية التدريب. وهذا يمنح القائمين على إعداد اللاعبين فرصة لبناء البرنامج التدريبي المباشر ومدعمات البرنامج التدريبي غير المباشرة. ويُشير (Rodrigues et all)., 2016) أن علم التدريب الرياضي ليس حديث النشأة، إلا أن التطور المستمر في علم التدريب جعلت منه علماً ذا ثراء واسع يدعو الباحثين للاهتمام بمستجداته.

وتتمثّل الأهمية النظرية لهذه الدراسة لكونها الدراسة الأولى التي تهتم بتأطير العلوم الأساسية ذات الأولوية في لئي إعداد وتأهيل المدرب الرياضي على مستوى سلطنة عمان، كما أنها وبحسب اطلاع الباحثين أنها من الدراسات البحثية النادرة على مستوى الوطن العربي، وبالتالي فهي بمثابة اللبنة التي يستقي منها المهتمين والباحثين في مجال إعداد وتأهيل المدرب الرياضي والاستفادة منها في الإعداد العلمي الأكاديمي للمدربين أو من خلال الدورات التأهيلية المتخصّصة. وتطبيقياً ستُمكن هذه الدراسة المدربين الخبراء ومن في حكمهم من الاستفادة من نتائج هذه الدراسة في اختيار وتقييم المدرب الرياضي وفقاً للأطر العلمية التي ستفرزها نتائج هذه الدراسة، حيث يطمح الباحثين من خلال هذه الدراسة إلى إيجاد قاعدة مُوحدة تكون لها مرجعية لتحديد مدى أهلية المدرب الرياضي من قيادة اللاعبين في الألعاب الرياضية المختلفة، كما ستتيح هذه الدراسة مساحة جيدة لتكوين أو إعادة صياغة برامج التدريب الرياضي في أقسام التدريب الرياضي أو علوم الرياضة.

r-1 r- r مشكلة الدراسة

تتعكس جودة المعرفة التي يكتسبها المدرب في مهمة التدريب، لذا اتجهت كليات وأقسام التربية البدنية وعلوم

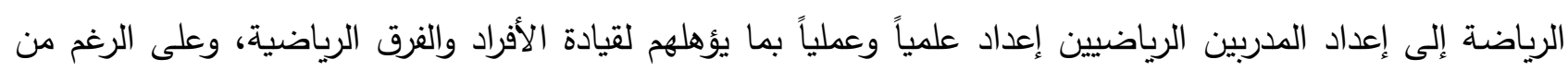
أهمية الإعداد الأكاديمي للمدرب الرياضي، يُشير محاي وعزيزي (Mohammadi \& Azizi), 2019) أن هناك القليل من البحوث التي أجريت لتحديد أولويات العلوم الأساسية التي يحتاجها المدرب وأن البحوث التي اهتمت باستقصاء

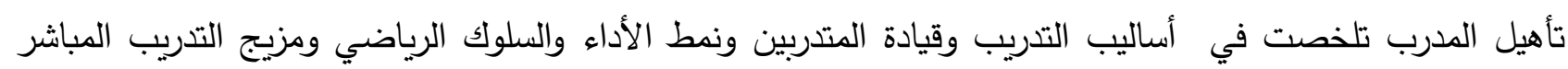
وغير المباشر. ويُشير ( ناش وآخرون Nash et all,.

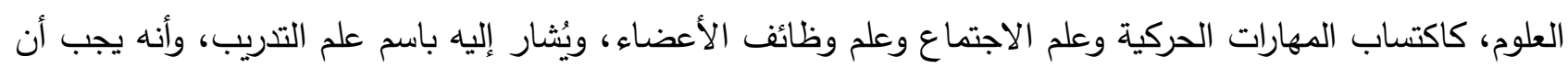

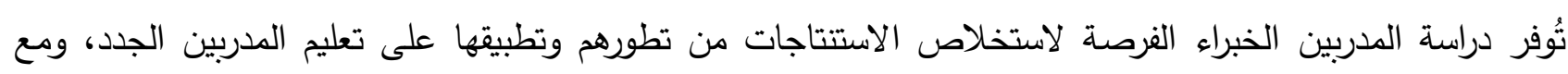
تتامي أقسام وكليات التربية البدنية وعلوم الرياضة من جهة، وتزايد الاقبال عليها من جهة أخرى، كان لابد من تحليل العلوم الأساسية للدراسات الجامعية في مرحلة البكالوريوس، وذلك لمعرفة العلوم التي تحتل المراتب المتقدمة ضمن التصن اهتمامات أقسام وكليات التربية البدنية وعلوم الرياضة، ليتم تسليط الضوه عليها عند صياغة برامج التدريب الرياضي. وقد لاحظ الباحثين من خلال تقصي الدراسات السابقة التي اهتمت بتطوير أداء المدربين أن هناك ندرة في تناول موضوع المدربين وآلية تطويرهم على المستوى الأكاديمي في مستوى الوطن العربي، ومن خلال اطلاع الباحثين لميدان التدريب الرياضي في سلطنة عمان - محل اهتمام هذه الدراسة- فإنه من المُلاحظ مع وجود المدربين المتخصّصين في الألعاب الرياضية المختلفة، إلا أنه يوجد عدد كبير من المدربين الذي يعتمدون في تدريبهم على خبرتهم التدريبية - استنساخاً- دون التحاقهم بدراسات أكاديمية تخصّصية أو دورات تأهيلية لتطوير مستواهم التدريبي، وهؤلاء المدربين هم ممن كانوا لاعبين أو هواه لتلك الرياضات. ولذا انبثقت هذه الدراسة لتسليط الاهتمام بالعلوم الأساسية 


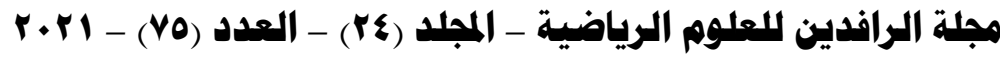

دراسة تحليلية للعلوم الأساسية ذات الأولوية في إعداد وتأهيل المدرب الرياضي

ذات الأولوية في إعداد وتأهيل المدرب الرياضي، وذلك باستقصاء وتفنيد العلوم الأساسية المُعتمدة في أقسام كليات التربية البدنية وعلوم الرياضة، فضلاً عن استقصاء حاجة المدربين ميدانياً لهذه العلوم وفقاً لأهميتها.

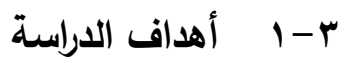
r-1-1 التعرف على العلوم الأساسية ذات الأولوية في إعداد وتطوير المدرب الرياضي من وجهة نظر مدربي الألعاب الرياضية المُختلفة وترتيبها؟ ب-1-r التعرف على العلوم الأساسية ذات الأولوية في إعداد وتطوير المدرب الرياضي وفقاً للمحتوى الدراسي المُعتمد لمرحلة البكالوريوس لدى الجامعات المُستهدفة. r-1-r التعرف على القضايا المُتعلقة بإعداد وتطوير المدرب الرياضي من وجهة نظر مدربي الألعاب الرياضية المُختلفة.

ץ-1-ـ التعرف على القضايا المُتعلقة بتطوير البيئة التدربيية من وجهة نظر مدربي الألعاب الرياضية المُختلفة. 1 - أسئلة الاراسة

تسعى هذه الدراسة للوصول إلى الأهداف من خلال الإجابة عن التساؤلات الأتية: ا-ـ-1 ما هي العلوم الأساسية ذات الأولوية في إعداد وتطوير المدرب الرياضي من وجهة نظر مدربي الألعاب الرياضية المُختلفة؟

1-ع - ما هي العلوم الأساسية ذات الأولوية في إعداد وتطوير المدرب الرياضي وفقاً للدحتوى الدراسي المُعتمد لمرحلة

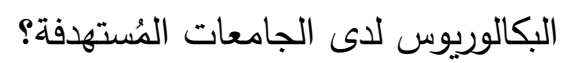

1-ـ - ما هي القضايا المُتعلقة بإعداد وتطوير المدرب الرياضي من وجهة نظر مدربي الألعاب الرياضية المُختلفة؟

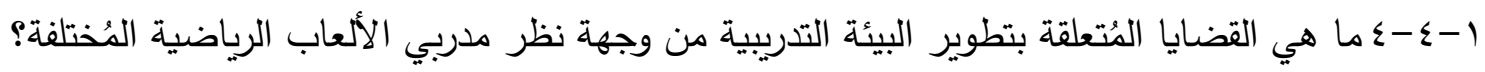

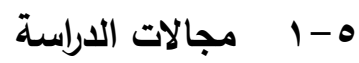
1-0-1 المجال البشري: طُبقت هذه الدراسة على مجموعة من مدربي الألعاب الرياضية المختلفة. 1-0-0 المجال المكاني: شملت هذه الدراسة مدربي الألعاب الرياضية المختلفة في سلطنة عمان.

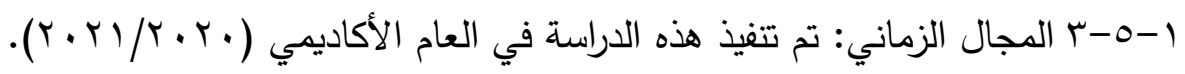

1- 1

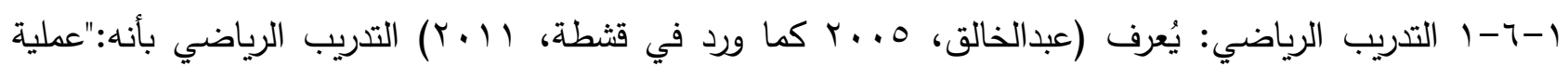
تربوية مخططة مبنية على الأسس العلمية والقواعد التربوية بهدف الوصول بالفرد لأعلى مستوى مدكن من النشاط البدني الممارس وذلك بتتمية قدرات الفرد البدنية ومهاراته الحركية وإمكاناته الخططية وقدراته العقلية وزيادة الدوافع النفسية

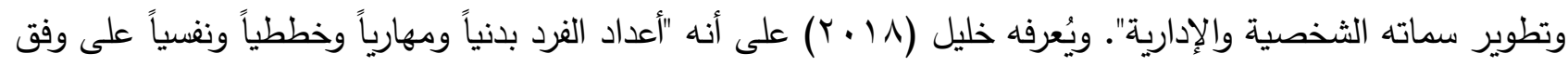
أسس ومبادئ علمية ولمدة زمنية مُعينة من أجل رفع كفاءته وقدرته في الوصول إلى المستويات العليا أو تحقيق الأرقام القياسية".

1-r-r المدرب الرياضي: تُعرف هذه الدراسة المدرب الرياضي بأنه: الفرد الذي يتولى قيادة المتدربين نحو تحقيق أهداف العملية التدربيية، وذلك برفع مستوى القدرات البدنية وتطوير الإمكانات المهارية والخططية، وتدعيم المواقف النفسية والتربوية والاجتماعية للمتدربين. (تعريف إجرائي). 


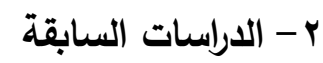

في دراسة تحجي وعزيزي (Mohammadi \& Azizi), 2019) حول تأثير الخصائص التعليمية للمدربين الرياضيين على قدراتهم دراسة حالة على المدربين الرياضيين في مدينة همدان الإيرانية. هدفت الدراسة لاستقصاء أولويات الخصائص التعليمية لمعلي الرياضة في محافظة حمدان. اعتمدت الدراسة على أداة استبانة طُبقت على عينة

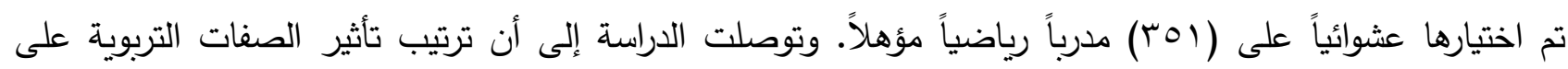

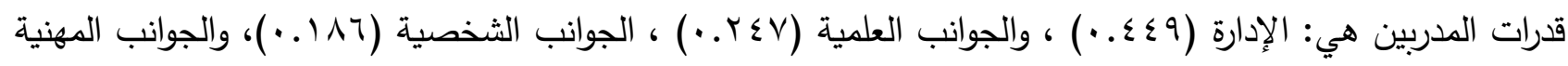

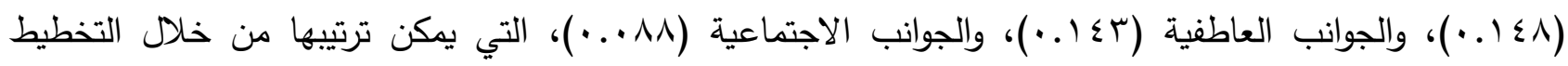
لمواجهة تحديات الخصائص التعليمية للمدربين الرياضيين مع إعطاء الأولوية للدورات التدربية قصيرة وطويلة المدى للإدارة والقيادة، وعلم النفس الرياضي والتكنولوجي والعلمي المتعلق بالمجال الرياضي لمحافظة همدان. وفي دراسة ريسفاندي وآخرون (Rizvandi et all., 2019) حول تقييم مؤشرات أداء المدربين لتطوير رياضة

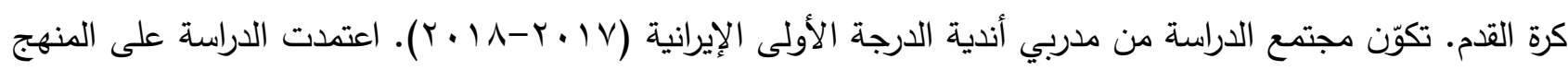
الوصفي التحليلي باستخدام أداة الاستبيان، وأظهرت الدراسة أن السلوك التدربي والمنافسة التدربية وثبات أداء الفريق الترائ

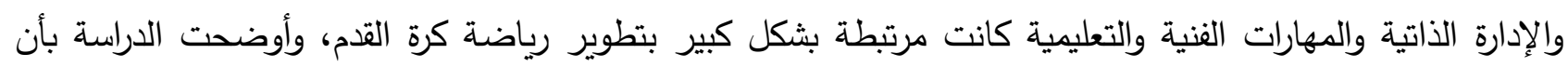
الخبرة لا تشكل عامل واضح لتطوير كرة القدم. وأوصت الدراسة بضرورة الاعتماد على هذه المؤشرات في اختيار المدربين.

وفي دراسة كارسون وآخرون (Carson et all,. 2018) حول الإناث في التدريب الرياضي: التحديات والتوتر وجودة الحياة. هدفت الدراسة إلى استقصاء التحديات التي تواجه الإناث في مجال التدريب الرياضي والتأثير الذي ممكن الممكن أن تحدثه هذه التحديات على جودة حياتهن. وقد حددت الدراسة مجموعة من التحديات تتعلق بالتوازن بين الحياة والعمل وطول فترة الابتعاد عن الأسرة، وحاجة الإناث لإثبات أنهنَّ أفضل من الذكور . وتوصلت الدراسة أن الإناث يواجهن نقص في الدعم المالي. وهذا ناتج بسبب الأيديولوجيا النوعية التقليدية التي قلصت من دور الإناث في مجال التدريب الرياضي.

وفي دراسة ستوسكوسكي وآخرون (Stoszkowski et all., 2016) حول المصادر والموضوعات واستخدام المعرفة من قبل المدربين. هدفت الدراسة إلى استكثاف تصورات المدربين لأساليبهم الفعلية والمفضلة لاكتساب معرفة تدريبية جديدة، وأنواع المعرفة التي يكتسبونها حاليًا أو يرغبون في اكتسابها، ومدى تطبيقهم للمعرفة الجديدة. شملت الدراسة (• بr) مدرباً جُمعت أراءهم عبر استطلاع الكتروني. وتوصلت الدراسة إلى أن المدربين يكتسبون المعرفة التدريبية من أنثطة التعلم غير الرسمية عن طريق التفاعل الاجتماعي مع الاعتماد بشكل نسبي على بعض المصادر الرسمية. وأثارت النتائج إلى ندرة استخدام المدربين للمعرفة المكتسبة في عملية التدريب، وأوصت الدراسة بوضع خطط تأهيل المدربين في مراكز التأهيل الرسمية. وفي دراسة آرايا وآخرون (Araya et all., 2015 ) حول مقتضيات تطوير المدرب: تصورات حول برنامج تعليم مدرب الدراسات العليا. هدفت الدراسة إلى استقصاء كيفية تطوير المدربين للمعرفة من خلال دورة تعليم المدربين ما بعد التخرج. واستقصاء تصورات المدربين للتغييرات التي أجروها على مواقفهم التدربية وسلوكياتهم ومهاراتهم وممارساتهم نتيجة لدراساتهم. اعتمدت الدراسة على المنهج الوصفي باستخدام المقابلة كأداة لجمع البيانات، تم تطبيقها 
على عينة شملت (V) مدرباً. وأظهرت النتائج أن المدربين طوروا معرفتهم من خلال مواقف التعلم ذات الصلة بسياق التدريب، وقد عززوا معرفتهم في بيئة تم إنشاؤها اجتماعيًا من خلال مجتمع الممارسة، وشعر المدربون أنهم أكثر استعدادًا لتطوير أداء الرياضيين نتيجة للمعرفة المكتسبة من خلال الدورة. وفي دراسة نيلسون وآخرون (Nelson et all.., 2013) حول تعزيز آلية تعلم المدربين: توصيات ممارسي

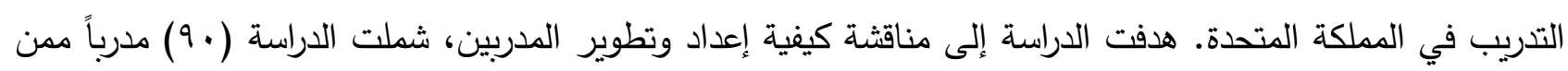
بلغت متوسط خبرتهم التدريبية (Tr) عاماً ومن ثماني رياضات، واستخدمت الدراسة المقابلة والاستبيان كأدوات لجمع

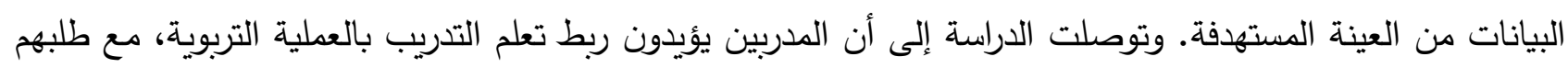
بتوفير مصادر تعلم متعددة وفرص للتوجيه في أثناء التعلم. وفي دراسة سوروجلال وآخرون (Surujlal., et all, معرفة أراء كل من (المدربين والإداريين الرياضيين) حول المدرب الرياضي في كل من دولة جنوب افريقيا وكندا، شملت العينة (•هr) من المدربين والإداريين من جنوب افريقيا، و (9 (7) من المدربين والإداريين من كندا، وتوصلت الدراسة إلى وجود فروق ذات دلالة إحصائية في الخبرة الثخصية والعلاقات العامة وسلوكيات المدربين بين البلدين، وأن هناك اختلاف بين آراء المدربين والإداريين في تقييم المدرب الرياضي. ومن المُلاحظ من خلال استعراض الدراسات السابقة اهتمام الباحثين بدراسة السلوك الإنساني والتفاعلات الاجتماعية والخبرة الثخصية والتصورات حول بيئة التدريب والمهارات الفنية للمدرب، وقد اعتمد الباحثين في دراساتهم على أداتين أساسيتين تمثلت في المقابلة والاستبيان، وقد بلغ متوسط العينات التي شاركت في الدراسات السابقة (9 ؟؟ب) مفحوصاً، وقد طُبقت الدراسات السابقة في بيئات مُختلفة شملت كل من آسيا وافريقيا وأوروبا. وقد استفادت هذه الدراسة مما توصلت إليه الدراسات السابقة في فهم بعض جوانب تطوير المدرب عبر المصادر الرسمية وغير الرسمية، وسعت هذه الدراسة لإضافة معلومات نوعية باستخدام أداة تحليل المحتوى الدراسي لمرحلة البكالوريوس في أقسام التدريب الرياضي (علوم الرياضة) في بعض الجامعات، وذلك لمعرفة العلوم الأساسية التي اعتمدتها الجامعات لإعداد وتأهيل المدربين. r- ب إجراءات الدراسة r-

اعتمدت هذه الدراسة على المنهج الوصفي التحليلي، وذلك لملائمته لأهداف هذه الدراسة، الذي يعتمد بشكل

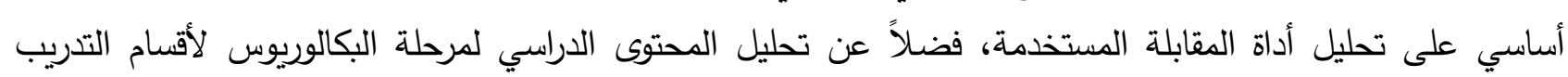
الرياضي (علوم الرياضة) في كل من الوطن العربي (جمهورية مصر العربية)، وأوروبا (المملكة الكُتحدة)، فهذا المنهج قائم أساساً على جمع المعلومات عن الظاهرة قيد الدراسة، ومن ثم تحليلها وتفسيرها للحصول على النتائج واستخلاص الاستنتاجات وإقرار التوصيات. r-r اعتمدت الدراسة على عيّنة تكوّنت من (7؟) مدرباً من مدربي الألعاب الرياضية المختلفة ومن محافظات 
اتبعت الدراسة سلسلة من الخطوات العلميّة، كالآتي:

ץ-r-1 دراسة الأدبيات السابقة التي أجريت على المستويات والإقليمي والعالمية والتي اهتمت بموضوع إعداد وتطوير المدرب الرياضي وبناء الإطار النظري للدراسة وتحديد منهجيتها.

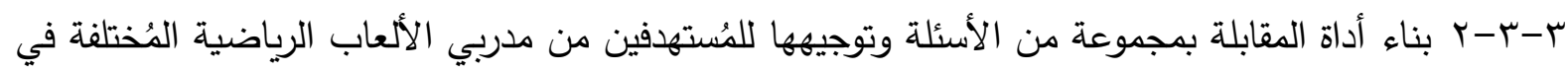

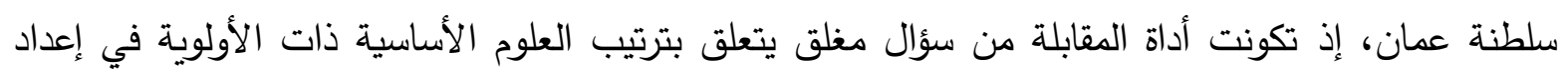
وتطوير المدرب الرياضي، وهي: أسس تدريب الرياضة للجميع، وأسس تدريب الرياضة النسائية، والرياضة

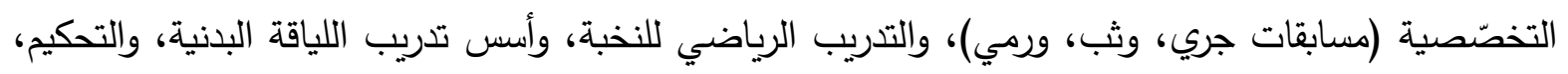
والإصابات الرياضية، وتغذية الرياضيين والمشروبات الرياضية، وعلم النفس الرياضي، والإدارة الرياضية. وسؤال مفتوح حول ذكُر القضايا المُتعلقة بتطوير وإعداد المدرب الرياضي. فضلاً عن السؤال مفتوح حول ذكُر القضايا المُتعلقة بتطوير البيئة التدريبية. ب-r-r ركزت الدراسة على تحليل المحتوى الدراسي لمرحلة البكالوريوس لأقسام التدريب الرياضي المُعتمدة في كل من الوطن العربي (جمهورية مصر العربية)، والجامعات هي: ( المنصورة، والإسكندرية، وكفر الثيخ، Sports ( وأسيوط، وأسوان، وبنها)، وكذلك تحليل المحتوى الدراسي لمرحلة البكالوريوس لأقسام علوم الرياضة (Science في المملكة المتحدة، وذلك بانتقاء أفضل (T) أقسام لعلوم الرياضة في جامعات المملكة المتحدة

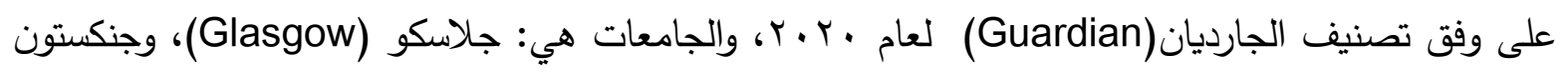
(Kingston)، ولافبرا (Loughborough)، و و برمنجهام (Birmingham)، و مانثستر ميتروبوليتان (Murham) (Manchester Metropolitan) r-r-ء جمع المحتوى الدراسي لتخصص التدريب الرياضي لمرحلة البكالوريوس في أقسام التدريب الرياضي (علوم الرياضة) في كل من جمهورية مصر العربية والمملكة المُتحدة. ז-r-ه تحليل أداتي الدُقابلة والمحتوى الدراسي لتخصص التدريب الرياضي لمرحلة البكالوريوس في أقسام التدريب الرياضي. r-r-r عرض نتائج أداتي المقابلة وتحليل المحتوى ومن ثم مناقشة وتقفير النتائج. r-r- التوصل للاستتتاجات وإقرار التوصيات. r- الأساليب الإحصائية اعتمدت الدراسة على برنامج الحزم الإحصائية للعلوم الاجتماعية (SPSS) لـعالجة البيانات، حيث تم استخدام نماذج الإحصاءات الوصفية (Descreptive): والتي تمثلت في استخدام النسب المئوية (Percentage) ، وهي من أنسب النماذج الإحصائية لوصف الظاهرة قيد الدراسة.

\section{ع - عرض ومناقشة النتائج}

ع-1 السؤال الأول: ما هي العلوم الأساسية ذات الأولوية في إعداد وتأهيل المدرب الرياضي من وجهة نظر مدربي الألعاب الرياضية المُختلفة؟ وللإجابة عن هذا السؤال اعتمدت الدراسة على تحليل أداة الئقابلة باستخدام النسب المئوية كما هو موضح في الثكل البياني (l) 


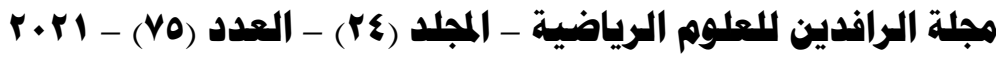

دراسة تحليلية للعلوم الأساسية ذات الأولوية في إعداد وتأهيل المدرب الرياضي

الثكل البياني (1)

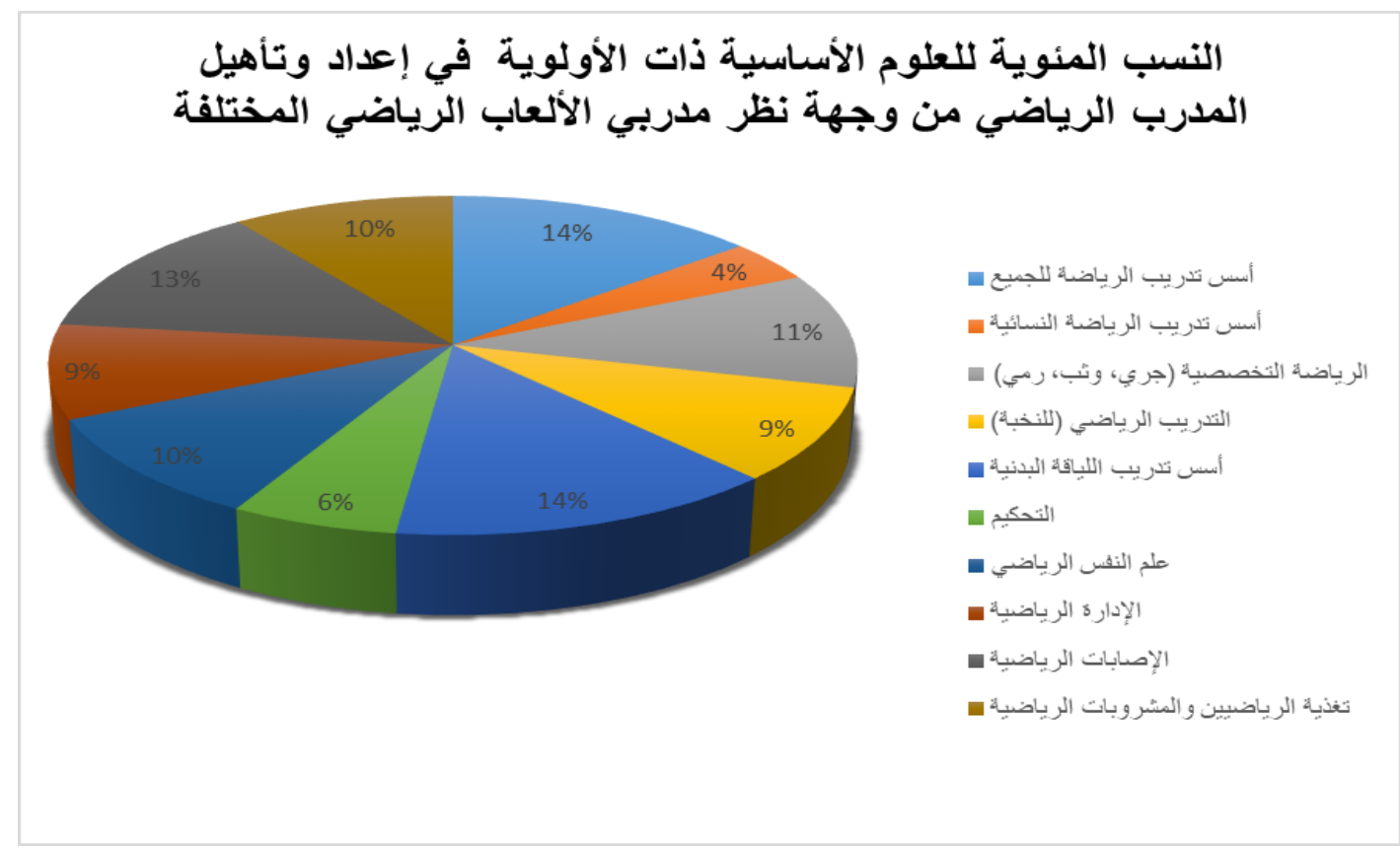

ويُلاحظ من خلال الثكل البياني ( ) أن أسس تدريب اللياقة البدنية وأسس تدريب الرياضة للجميع جاءت في

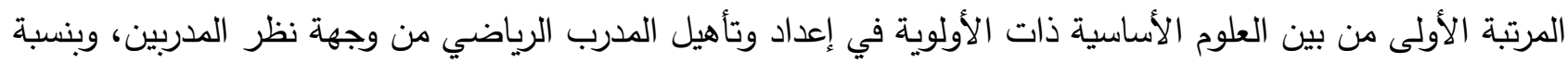

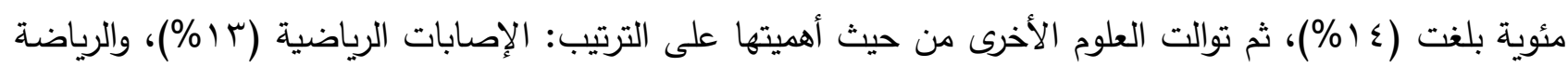

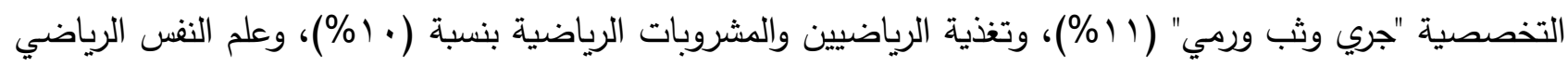

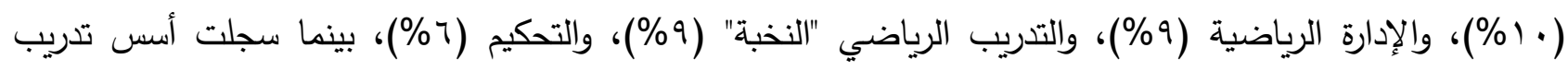

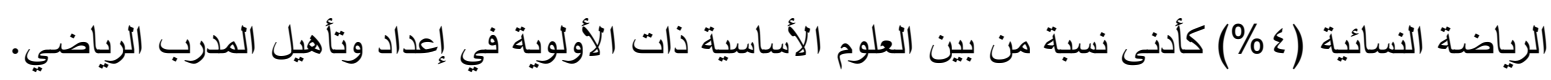

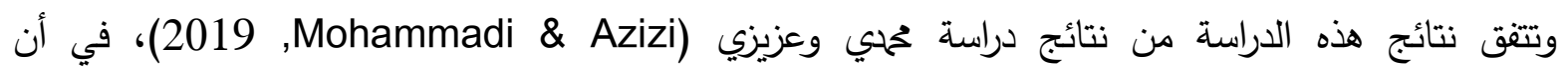
الخصائص العلمية تمثل أولوية بالنسبة للمدرب الرياضي. وتتثق نتائج هذه الدراسة مع نتائج دراسة كارسون (وآخرون 2018 (Carson et all)

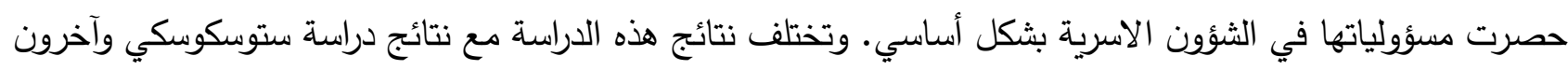
Stoszkowski et all)., 2016)، التي أظهرت أن المدربين نادراً ما يعتمدون على المعرفة المكتسبة لأداء مهامهم التدريبية.

ويُعسر الباحثين سبب إدراك الددربين لأهمية الجوانب العلمية في التدريب الرياضي في أن نجاح عملية التدريب

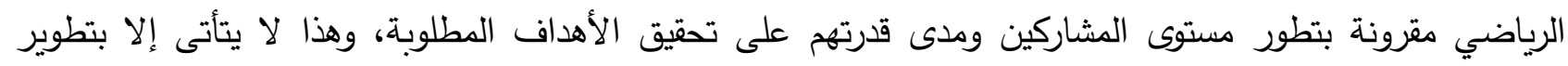

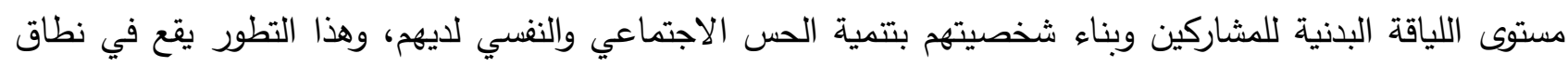

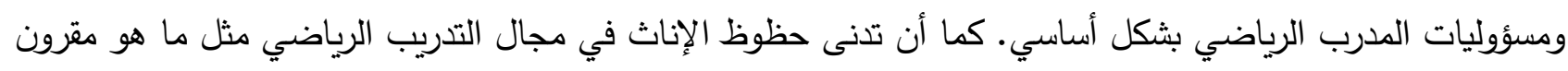

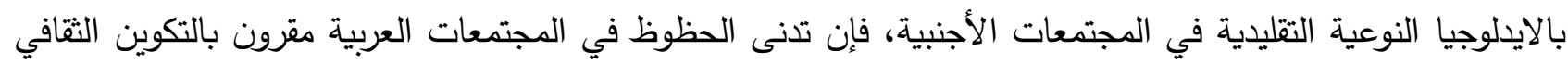

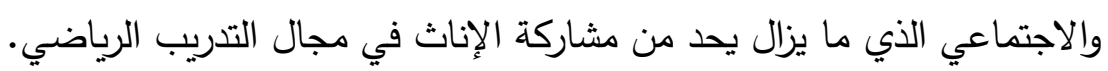




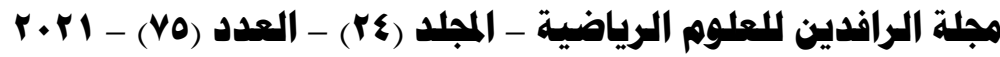

دراسة تحليلية للعلوم الأساسية ذات الأولوية في إعداد وتأهيل المدرب الرياضي

ع - ب السؤال الثاني: ما هي العلوم الأساسية ذات الأولوية في إعداد وتطوير المدرب الرياضي وفقاً للمحتوى

الدراسي المُعتمد لمرحلة البكالوريوس لدى الجامعات المُستهدفة؟ ولإجابة عن هذا السؤال اعتمدت الدراسة تحليل البرامج الدراسية لمرحلة البكالوريوس لمجموعة من الجامعات المُستهدفة في مجموعتين منفصلتين

وهما: أقسام التدريب الرياضي في كليات التربية الرياضية جمهورية مصر العربية، وأقسام علوم الرياضة بجامعات المملكة المتحدة.

أولاً: برامج التدريب الرياضي في أقسام التدريب الرياضي بكليات التربية الرياضية في جمهورية مصر العربية: وقد اعتمدت الدراسة على حصر برامج التدريب الرياضي المُعتمد في الخطة الدراسية لأقسام التدريب الرياضي، دون

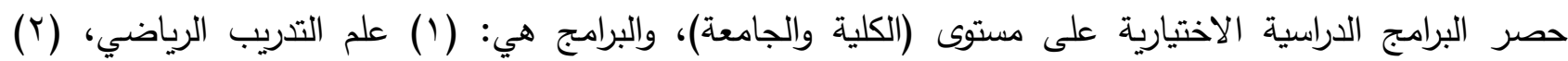

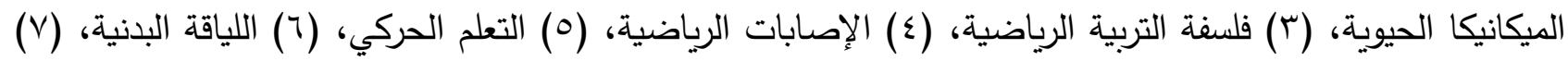
تدريب رياضة التخصص، (^) فسيولوجيا الرياضة، (9) أسس انتقاء الناشئين، (• (1) القياس والتقويم في التدريب

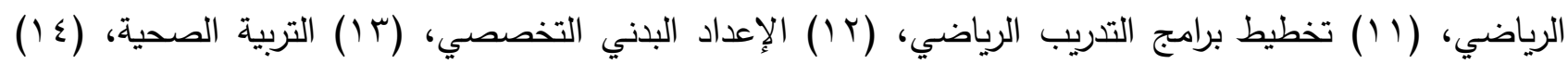
سيكولوجية التدريب الرياضي، (0) مبادئ تدريب الناشئين، (1 إل) الإعداد الفني والخططي، (IV) تحليل الأداء الحركي، (1) (1) تحكيم رياضة التخصص، (9 (1) الترويح وأوقات الفراغ، (·r) التغذية للرياضيين، (Y) تكنولوجيا

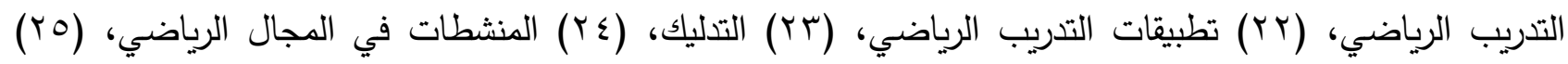
الإدارة الرياضية. ويُبين الثكل البياني (r) حجم برامج التدريب الرياضي في أقسام التدريب الرياضي بكليات التربية الرياضية في جامعات جمهورية مصر العربية.

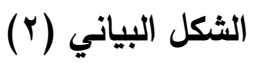

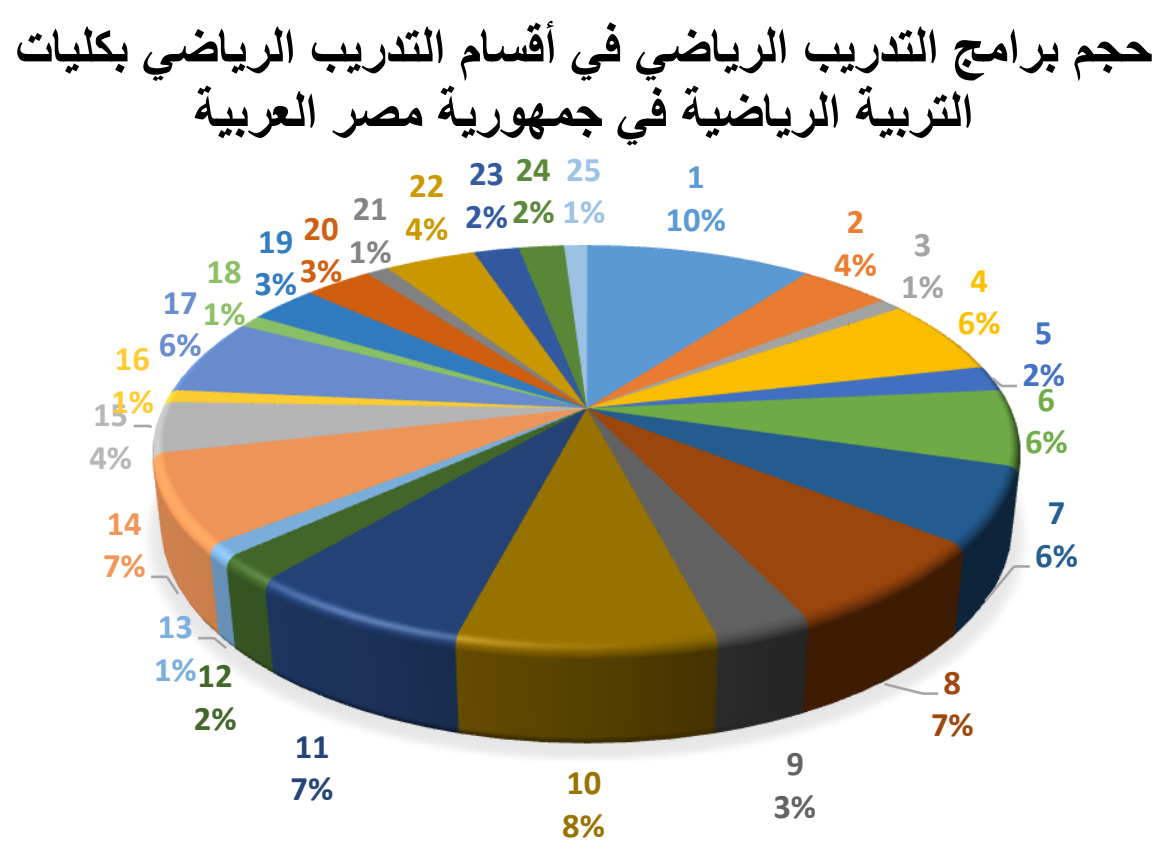


ويُلاحظ من خلال الرسم البياني (r) أن العلوم الأساسية ذات الأولوية في إعداد وتأهيل المدرب الرياضي في أقسام التدريب الرياضي بجامعات جمهورية مصر العربية هي: علم التدريب الرياضي وبنسبة (· (1\%)، ثم القياس والتقويم في التدريب الرياضي وبنسبة (^٪\%)، ثم احتلت العلوم (فسيولوجيا الرياضة، تخطيط برامج التدريب الرياضي، سيكولوجية التدريب الرياضي) في المرتبة الثالثة وبنسبة (v\%)، بينما سجلت علوم (فلسفة التربية الرياضية، والتربية الصحية، وتكنولوجيا التدريب الرياضي، الإدارة الرياضية) أدنى نسبة من بين العلوم الأخرى حيث بلغت (1) (1\%). ثانياً: برامج التدريب الرياضي في أقسام علوم الرياضة بجامعات المملكة المتحدة: وقد اعتمدت الدراسة الإدانة

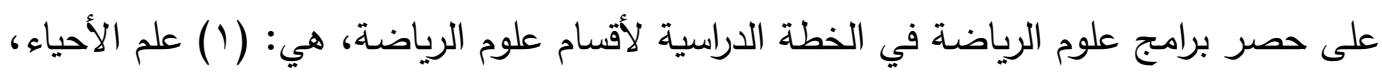

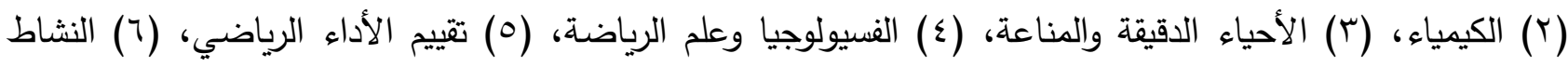

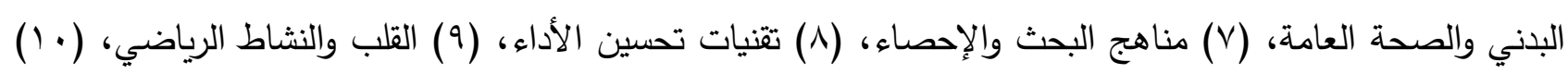

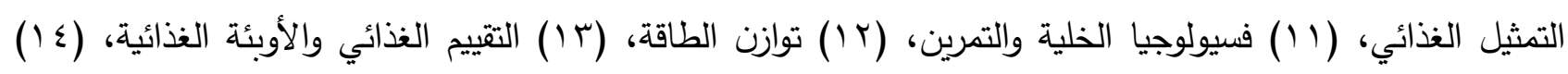

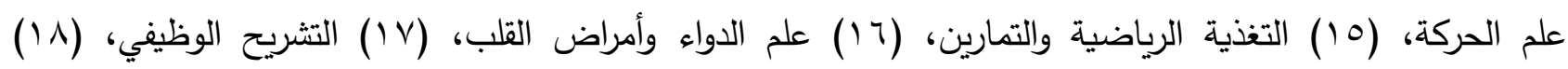

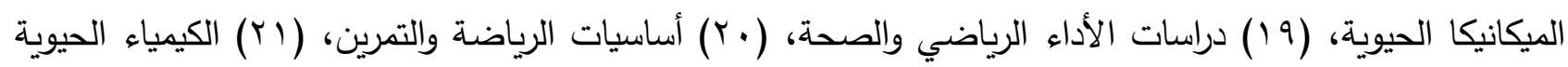

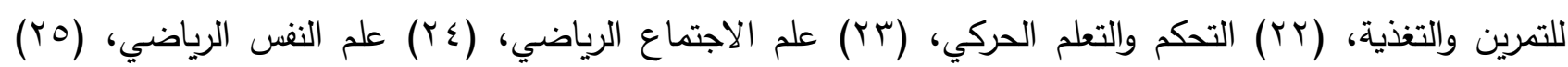
صناعة الرياضة. ويُبين الثكل البياني (r) حجم برامج التدريب الرياضي في أقسام علوم الرياضة بجامعات المملكة المتحدة.

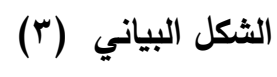

\section{حجم برامج علوم الرياضة في أقسام علوم الرياضة بجامعات المملكةة المُتحدة}

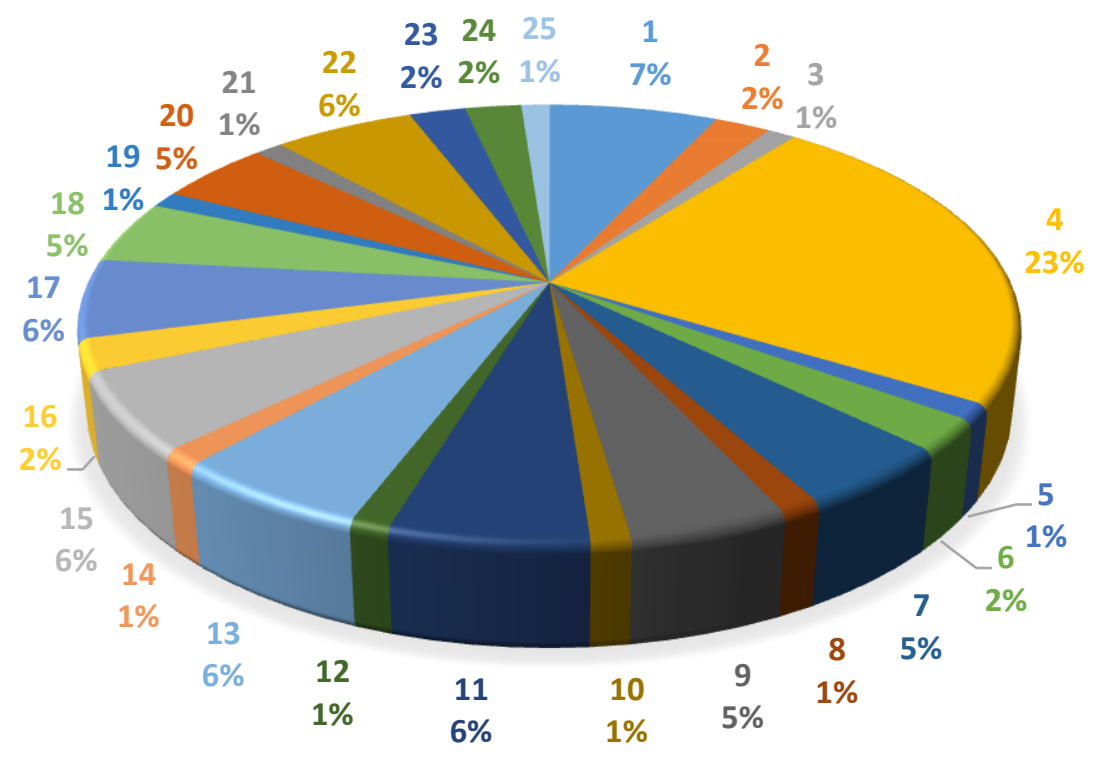


ويُلاحظ من خلال الرسم البياني (r) أن العلوم الأساسية ذات الأولوية في إعداد وتأهيل المدرب الرياضي في

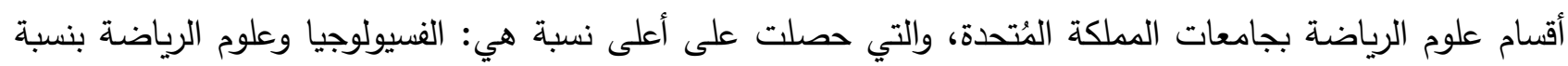

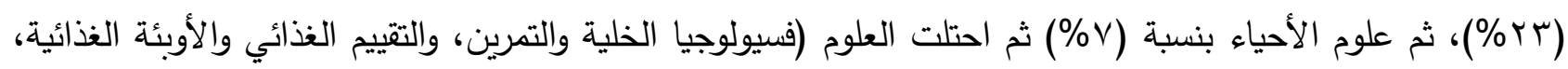
التغذية الرياضية والتمارين، التشريح الوظيفي، التحكم والتعلم الحركي)، المرتبة الثالثة من بين العلوم الأساسية ذات الأولوية في إعداد وتأهيل المدربين الرياضيين وبنسبة (7 \%)، بينما بلغت أدنى نسبة للعلوم ذات الأولوية في إعداد وتأهيل المدرب الرياضي (1\%) في كلٌ من (الأحياء الدقيقة والمناعة، وتقييم الأداء الرياضي، ولاضين وتقنيات تحسين الأداء، والتمثيل الغذائي، وتوازن الطاقة، وعلم الحركة، ودراسات الأداء الرياضي والصحة، والهيه والكيمياء الحيوية للتمرين والتغذية، وصناعة الرياضة). ويُلاحظ الباحثين من خلال استعراض برامج التدريب الرياضي في كل من أقسام التدريب الرياضي في كليات التربية الرياضية بجمهورية مصر العربية، وأقسام علوم الرياضة بجامعات المملكة المُتحدة أن هناك اختلاف في العلوم الأساسية ذات الأولوية في إعداد وتأهيل المدرب الرياضي، ويعزو الباحثين ذلك بأن أقسام التدريب الرياضي بكليات التربية الرباضية في جامعات جمهورية مصر العربية تقع في نطاق العلوم التربوية، مما يلزم طلبة أقسام التدريب

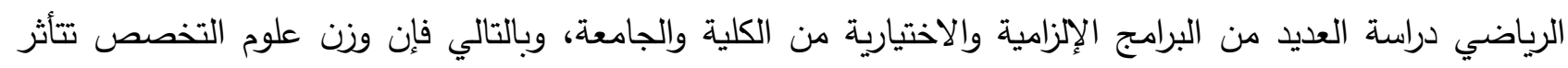
بحصة البرامج الإلزامية والاختيارية من الخطة الدراسية لمرحلة البكالوريوس، في المُقابل فإن أقسام علوم الرياضة بجامعات المملكة المُتحدة مستقلة بحد ذاتها، وبالتالي فإن البرامج الدراسية في الخطة الدراسية لمرحلة البكالوريوس تتركز في علوم الرياضة فقط سواء كانت برامج إلزامية أم اختيارية. ع - السؤال الثالث: ما هي القضايا المُتعلقة بإعداد وتطوير المدرب الرياضي من وجهة نظر مدربي الألعاب الرياضية المُختلفة؟ وللإجابة عن هذا السؤال، فقد فندات الدراسة استجابات العينة المُستهدفة على أداة المُعابلة. وقد تركزت استجابات العينة في مجموعة من القضايا، وهي: - التقريخ للمشاركة بالدورات والدراسات التأهيلية، والاهتمام بزيادة عددها لتأهيل الكوادر البشرية.

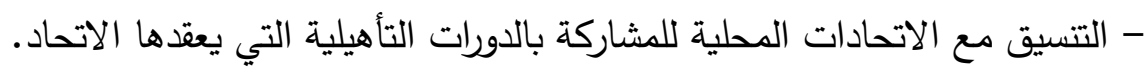
- الاهتمام وتقديم الفرصة لجميع المدربين للمشاركة بالدورات والدراسات التأهيلية. - استمرار وتكثيف الدورات والبرامج العلمية الرياضية. - تقديم الدورة التأسيسية للمدربين قبل البطولة بأشهر حتى يتم الاستفادة من المادة العلمية المطروحة في الدورة. - عمل الدورات التدريبية في الألعاب الرباضية كافة. - عمل دورات تأهيلية طويلة الأمد لتحقيق أكبر استفادة. ع - ء السؤال الرابع: ما هي القضايا المُتعلقة بتطوير البيئة التدرببية من وجهة نظر مدربي الألعاب الرياضية المُختلفة؟ وللإجابة على هذا السؤال، فقد فندت الدراسة استجابات العينة المُستهدفة على أداة المُقابلة. وقد تركزت استجابات العينة في مجموعة من القضايا، وهي: - إنثاء مرافق رياضية حديثة تُسهم في تتمية قدرات الأفراد والجهاز التدريبي. - توفير أسس الإسعافات الأولية في المرافق الرياضية. - عمل المسابقات بشكل مستمر لتطوير أداء المشاركين والجهاز الفني. 
- التثريغ والاهتمام بالبرامج الرياضية بعد ساعات الدوام الرسمية. - تفريغ اللاعبين لفترة مناسبة وذلك للاستعداد للبطولات. ه- - الاستنتاجات والتوصيات ه-1 الاستتتاجات

- احتلت أسس تدريب اللياقة البدنية وأسس تدريب الرياضة للجميع في مقدمة العلوم الأساسية ذات الأولوية في إعداد وتأهيل المدرب الرياضي من وجهة نظر مدربي الألعاب الرياضية المُختلفة. -استقلالية أقسام التدربب الرياضي عن العلوم التربوية في جامعات المملكة المُتحدة، أوجدت اختلافاً في العلوم الأساسية ذات الأولوية في إعداد وتأهيل المدرب الرياضي مقارنة بالعلوم الأساسية لإعداد وتأهيل المدرب

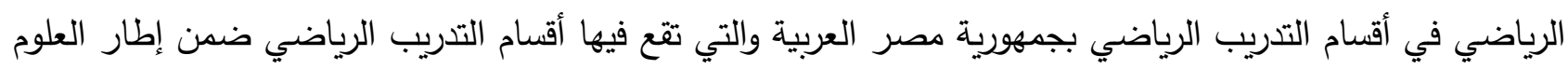
التربوية. - من أهم القضايا المتعلقة بإعداد وتأهيل المدرب الرياضي من وجهة نظر مدربي الألعاب الرياضية المُختلفة هي الاهتمام بتفريخ المشاركين في المناسبات الرياضية وتأهيل مرافق المنشآت الرياضية بشكل يُتيح للمدربين والمشاركين ممارسة مهامهم وأدوارهم بما يحقق أهداف البرنامج التدريبي. r-0 - ضرورة مواءمة احتياجات المدربين للألعاب الرياضية المختلفة مع برامج إعداد وتأهيل المدربين في أقسام التدريب الرياضي في الدراسات الجامعية. - ضرورة إيجاد معايير مُوحدة لبناء برامج التدريب الرياضي في أقسام وكليات التربية الرياضية لاسيما في الوطن العربي، ودعمها بالمعايير الدولية. - ضرورة تأهيل وبناء المُنشآت الرياضية بما يتوافق مع المعايير الحديثة لتُههم في تلبية احتياجات المدربين والمشاركين.

\section{المصادر}

ا. الحاوي، يحيى السيد .(r . . r). المدرب الرياضي بين الأسلوب التقليدي والتقنية الحديثة في مجال التدريب.

$$
\text { المركز العربي للنشر: جمهورية مصر العربية. }
$$

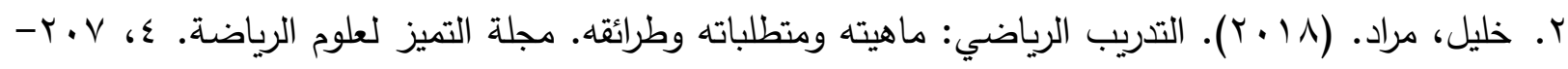

TYS

r. قشطة، عمر نصر الله ـ(11 + (1). المدرب الرياضي من خلال معايير الجودة الثاملة. دار الوفاء لدنيا الطباعة

$$
\text { والنشر ، الإسكندرية: جمهورية مصر العربية. }
$$

4. Surujlal, J., Singh, P. C., \& MacLean, J. (2009). Performance appraisal of coaches: a comparative study management. African Journal for Physical Health Education, Recreation and Dance, 15(1), 74-90. 


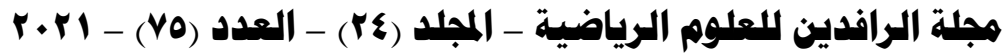 \\ دراسة تحليلية للعلوم الأساسية ذات الأولوية في إعداد وتأهيل المدرب الرياضي}

5. Nelson, L., Cushion, C., \& Potrac, P. (2013). Enhancing the provision of coach education: The recommendations of UK coaching practitioners. Physical Education and Sport Pedagogy, 18(2), 204-218.

6. Stoszkowski, J., \& Collins, D. (2016). Sources, topics and use of knowledge by coaches. Journal of sports sciences, 34(9), 794-802.

7. Rizvandi, A., Taghipour Gharbi, M., Esmaeili, M., \& Ashraf Ganjoee, F. (2019). The Evaluation of Performance Indicators of Coaches in Football Development. Journal of Humanities Insights, 3(04), 248-254.

8. Cruickshank, A., \& Collins, D. (2015). The sport coach. Leadership in sport, 155-172. Araya, J., Bennie, A., \& O'Connor, D. (2015). Understanding performance coach development: Perceptions about a postgraduate coach education program. International Sport Coaching Journal, 2(1), 3-14.

9. Lara-Bercial, S., North, J., Petrovic, L., Oltmanns, K., Minkhorst, J., Hämäläinen, K., \& Livingstone, K. (2017). European Sport Coaching Framework v1.

10. Rodrigues Marques, R. F., Nunomura, M., \& Pombo Menezes, R. (2016). Sports coaching science in Brazil.

11. Carson, F., McCormack, C., \& Walsh, J. (2018). Women in sport coaching: Challenges, stress and wellbeing. ACTIVE: Journal of Physical Education, Sport, Health and Recreation, 7(2), 63-67.

12. Nash, C., \& Collins, D. (2006). Tacit knowledge in expert coaching: Science or art?. Quest, 58(4), 465-477.

13. Mohammadi, M., \& Azizi, B. (2019). Determining the effect of educational characteristics of sports coaches in their ability (a case study of sport coaches in Hamadan Province), Humanidades \& Inovação, 6(13), 77-88.

14. Gano-Overway, L., Van Mullem, P., Long, M., Thompson, M., Benham, B., Bolger, C., ... \& Schuster, D. (2020). Revising the national standards for sport coaches within the USA. International Sport Coaching Journal, 7(1), 89-94.

15. Taylor, B., \& Garratt, D. (2010). The professionalisation of sports coaching: Definitions, challenges and critique. Sports coaching: Professionalisation and practice, 99-117. 